\title{
CIDADES SUSTENTÁVEIS E CIDADES INTELIGENTES: UMA ANÁLISE DOS RANKINGS ARCADIS E EUROPEAN SMART CITIES
}

\author{
Luiz Eduardo Brand Flores ${ }^{1}$, Clarissa Stefani Teixeira ${ }^{2}$ \\ ${ }^{1}$ Universidade Federal do Estado de Santa Catarina (UFSC) \\ ${ }^{2}$ Engenharia de Gestão do Conhecimento (EGC) \\ ${ }^{1}$ luizebf@hotmail.com \\ ${ }^{2}$ clastefani@gmail.com
}

\section{Resumo}

O presente estudo buscou relacionar e analisar dois rankings de cidades: um de cidades inteligentes e outro de cidades sustentáveis. Para melhor entendimento, conceitos de cidades inteligentes e cidades sustentáveis foram explorados e explanados. Os rankings analisados pelo estudo foram: Arcadis Sustainable Cities Index 2016 para cidades sustentáveis e European Smart Cities para cidades inteligentes. A partir deles, as dimensões e os indicadores de cada um foram descritos e comparados para esclarecer as semelhanças e as diferenças entre eles. Por fim notou-se que os rankings possuem semelhanças, entretanto divergem em seus focos.

Palavras Chave: Ranking, Cidade, Inteligente, Sustentabilidade.

\section{SUSTAINABLE CITIES AND SMART CITIES: AN ANALYSIS OF ARCADIS RANKINGS AND EUROPEAN SMART CITIES}

\begin{abstract}
The present study sought to relate and analyze two city rankings: one of smart cities and other of sustainable cities. For better understanding, concepts of smart cities and sustainable cities were explored and explained. The rankings analyzed by the study were: Arcadis Sustainable Cities Index 2016 for sustainable cities and European Smart Cities for smart cities. Through them, the dimensions and the indicators of each one were described and compared to clarify the similarities and as differences between them. Finally it was noticed that the rankings have similarities, however they diverge in their foci.
\end{abstract}

Keywords: Ranking, City, Smart, Sustainability.

\section{Introdução}

Organismos vivos precisam de comida, água e oxigênio para que assim possam convertê-los em energia. Essa energia gerada pode, agora, ser empregada de forma a desenvolver alguma atividade que o mesmo necessite como: andar, respirar, pensar. Entretanto parte deste processo se transforma em resíduo ou restos. Esta é a síntese do metabolismo de inúmeros organismos (SCIENCE FOR ENVIRONMENT POLICY, 2015). Na mesma linha de raciocínio, cidades têm a mesma tendência metabólica, ou seja, precisam de água, matérias primas e processos para que se gere energia, dando o que é necessário para seus habitantes e por fim liberam resíduos (KENNEDY; CUDDIHY; ENGEL-YAN, 2007). Contudo, a população urbana passa dos $50 \%$ da população mundial [UN, 2014] que em 
conjunto com o crescimento desenfreado da indústria causa uma maior destruição do meio ambiente, oferecendo riscos a atividades econômicas e a saúde em geral (ALBINO; BERARDI; DANGELICO, 2015).

Em virtude destas problemáticas, questões relacionadas ao modo como as cidades se organizam vêm sendo levantadas, visando transformá-las em mais sustentáveis e mais inteligentes (BULKELEY; BETSILL, 2005; EUROPEAN COMMISSION, 2003; GIFFINGER; HAINDL, 2007). Logo, as cidades ao redor do mundo mostram crescente interesse por sustentabilidade, i.e. desenvolver-se sem ultrapassar as capacidades de regeneração da biosfera (CARRETERO, 2002; GOODLAND; DALY, 1996; HIREMATH et al., 2013; HOLDEN; LINNERUD; BANISTER, 2017). Recentemente a discussão vem alterando ligeiramente o foco para: como a sustentabilidade pode ser alcançada com a ajuda da "inteligência", ou seja, tecnologia e conectividade, envolvendo todos os sistemas da região urbana [ALBINO; BERARDI; DANGELICO, 2015; AHVENNIEMI et al., 2017].

A partir destes novos conceitos de cidades, informações, iniciativas e investimentos promovem ações na intenção de evoluir os meios urbanos. Estes incentivos se tornaram cada vez mais relevantes, volumosos e efetivos (JESSOP, 2000). Como resposta e forma de controlar e medir estes incentivos, surgiram os rankings, que consistem em vários indicadores que medem a performance dos objetos em um aspecto do atributo (MEIJERING; KERN; TOBI, 2014). Estes surgiram em grande quantidade, com o propósito de apontar as melhores cidades dos devidos temas e descrever pontos fortes e pontos fracos das mesmas. Todavia, por mais parecidos que sejam, cada um deles carrega um método de avaliação variando dimensões, indicadores e pesos entre os indicadores, o que retrata uma oscilação entre os ranqueados (GIFFINGER; HAINDL, 2007). Portanto, o objetivo deste artigo é analisar os rankings de cidades inteligentes e cidades sustentáveis de forma a identificar convergências entre indicadores.

\section{Metodologia}

O estudo é considerado como sendo descritivo exploratório (GIL, 2002) e busca analisar os rankings de cidades inteligentes e sustentáveis de forma a identificar convergência entre indicadores. Inicialmente, foram definidos os conceitos para os dois tipos de cidades. Especificamente tratando dos rankings, o presente estudo analisou o maior ranking dedicado a classificar cidades inteligentes: European Smart Cities e, também um dos rankings de maior relevância no quesito classificação de cidades sustentáveis: Arcadis Sustainable Cities Index 2016 afim de compreender cada dimensão e indicador considerado pelo ranking. Para tanto, foram consideradas as publicações " Smart Cities Ranking: an Effective Instrument for the Positioning of Cities?" do European Smart Cities e " Sustainable Cities Index 2016 Global Web" de Arcadis Sustainable Cities Index 2016, disponibilizadas em suas respectivas páginas.

Assim, foram identificadas as relações entre os rankings e entre as classificações de cidades de cada um. As análises foram realizadas de forma qualitativa organizando os indicadores dos rankings com propósitos de medidas semelhantes em um mesmo conjunto de análise.

\section{Conceitos}

\subsection{Cidade Inteligente}

Não há uma definição única para "Smart Cities", o que se tem são muitos pontos de vista diferentes, assim cidade inteligente tem muitas variações em seu conceito. Além disso, uma gama de variações conceituais pode ser obtida alterando "smart" por outros adjetivos, 
como "digital". Segundo Albino [2015], o termo cidade inteligente primeiramente usado nos anos 1990 e tinha como foco tecnologias de comunicação e informação para que assim a cidade se definisse como inteligente. Entretanto, os conceitos e definições sobre as cidades inteligentes foram evoluindo e abrangendo mais dimensões para que a cidade fosse mais circular, sustentável, popular e conectada. De acordo com Geffinger [2007], existem seis dimensões numa cidade inteligente: economia, mobilidade, governança, meio ambiente, convívio e pessoas.

Uma cidade inteligente incorpora infraestrutura para facilitar a mobilidade, adicionar efetividade nos diversos setores, economizar energia, melhorar e preservar a qualidade do ar e da água, identificar problemas e consertá-los com rapidez, entre outras diversas qualidades. Estas operações são instrumentadas e guiadas sempre com conectividade entre os setores e sistemas (KANTER; LITOW, 2009).

\subsection{Cidade Sustentável}

A noção de sustentabilidade surgiu quando se notou um risco, ao meio ambiente, engatilhado perante o desenvolvimento de um paradigma social, econômico e urbano. $\mathrm{O}$ conceito surgiu e se tornou internacionalmente disseminado no final da década de 1980, seguido pela ampla adoção de estratégias e por abundantes discursos relacionadas ao tema (ELIAS; KROGSTIE, 2017). Hoje, o desenvolvimento sustentável pode ser definido como "Desenvolvimento que atende as necessidades do presente sem comprometer a capacidade das gerações futuras de atender às suas próprias necessidades." [BRUNDTLAND, 1987, p.41, tradução nossa].

A cidade sustentável tem como princípios melhorar o bem estar dos cidadãos e da sociedade como um todo através da integração do planejamento e da administração do meio urbano que aproveita os benefícios dos sistemas ecológicos protegendo e nutrindo esses recursos para gerações futuras (SUZUKI et al., 2010). Além disso, uma comunidade urbana sustentável envolve o entendimento da conexão da economia, da sociedade e do meio ambiente. Esta precisa promover a igualdade e inclusão social, ser economicamente produtiva, ter suas construções em harmonia com a natureza, preservar raízes históricas e ser mantida para todas as gerações. Uma comunidade não sustentável consome seus recursos mais rápido do que eles podem ser renovados e produz mais resíduo do que o sistema natural pode degradar (RAI, 2012).

\section{Rankings}

\subsection{Ranking European Smart Cities}

Pela grande variedade de conceitos de cidades inteligentes, vindo dos mais integrados à tecnologia, até os mais ligados a vertente da sustentabilidade, os rankings ganharam visibilidade. Por um lado, a comparação entre cidade ajuda investidores, além de ser um importante guia para as cidades julgarem seus pontos fortes e fracos, assim determinando metas e estratégias para o futuro (HIREMATH et al., 2013).

Perante a variedade de rankings, o grupo TU WIEN estudou as características básicas de rankings nacionais e internacionais, como indicadores, métodos de avaliação e potenciais benefícios dos mesmos e desenvolveu o próprio ranking, o European Smart Cities. Não só feito para mostrar as posições das cidades dentro do ranking, a iniciativa busca mostrar características das cidades para base de discussão estratégica, demonstrando que rankings são uma ferramenta significativa e eficiente na condução econômica, social e nos processos de cidades (NAM; PARDO, 2011). 
Conforme o ranking European Smart Cities [2014] uma cidade inteligente é uma cidade que atua em seis campos-chave do desenvolvimento urbano, construído sobre a combinação "inteligente" de doações e atividades de cidadãos autodeterminados, independentes e conscientes. Estes seis campos-chave são as seis dimensões do ranking e dentro destas existem os indicadores, conforme mostrado no Quadro 1. As dimensões do ranking são: economia inteligente, mobilidade inteligente, meio ambiente inteligente, pessoas inteligentes, convivência inteligente, governança inteligente.

Quadro 1: Dimensões, indicadores e, entre parênteses, pesos dos indicadores do ranking European Smart Cities

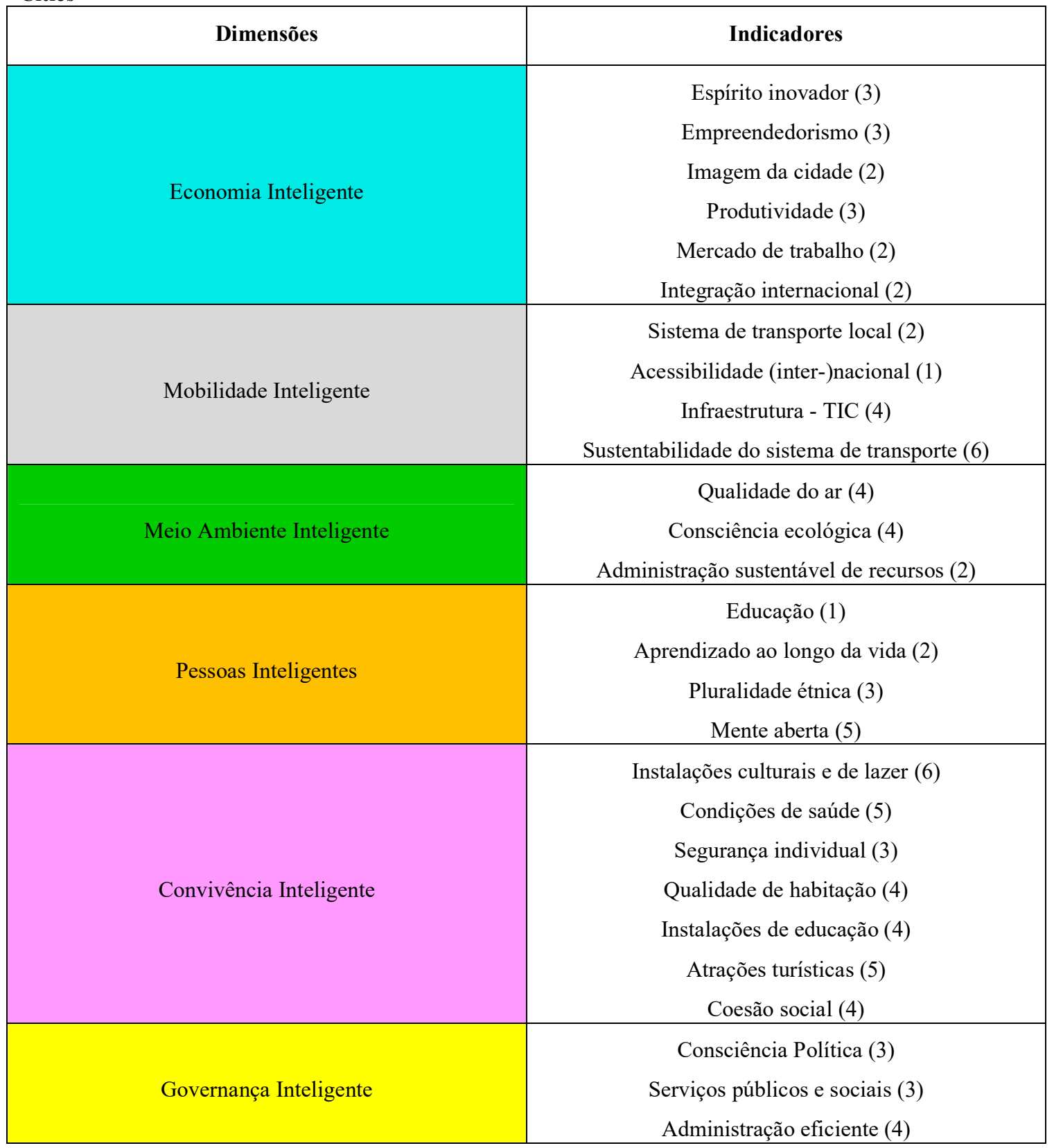

Fonte: http://www.smart-cities.eu/?cid=2\&ver $=4$ 
Os indicadores são definidos de diferentes maneiras e, assim, mostram níveis de valores diferentes que não podem ser mesclados de qualquer forma. Portanto, esses indicadores são padronizados através de uma transformação $\mathrm{z}$, resultando em uma distribuição com um valor médio ' 0 ' e um desvio padrão de ' 1 '. Através desta transformação, os indicadores são comparáveis e apropriados para qualquer procedimento de agregação (GIFFINGER; GUDRUN, 2010).

Cada dimensão apresenta seu próprio ranking e é a partir dos resultados das dimensões que as colocações do ranking geral são definidas.

\subsection{Ranking Sustainable Cities Index 2016}

Ações de sustentabilidade em cidades estão sempre inovando. E o acompanhamento e o relato dessas ações pode ajudar setores e indicadores importantes, como: avaliar o progresso da sustentabilidade urbana, identificar interações de apoio mútuo entre os pilares da sustentabilidade, expor os pontos fortes e fracos da cidade, estimular as cidades a participar da coleta sistemática de dados e etc (TELOS; DAGEVOS; ZOETEMAN, 2015).

A sustentabilidade não envolve mais, apenas, o meio-ambiente, envolve também os desafios do dia-a-dia dos cidadãos, ou seja, que o aumento dos estresses com a escassez de água, as mudanças climáticas, os preços da habitação e os fatores de emprego estão afetando a qualidade de vida. Assim, o ranking Arcadis de cidades sustentáveis considera a dimensão das pessoas dentro da experiência urbana (KUHLMAN; FARRINGTON, 2010).

De acordo com Batten [2016] o objetivo do primeiro Índice de Cidades Sustentáveis era levar 50 das cidades mais sobressalentes do mundo e analisar sua viabilidade como lugares para viver, seu impacto ambiental, sua estabilidade financeira e como esses elementos se complementam. Para o ranking de 2016, analisado por este trabalho, criou-se uma base de dados mais robusta para promover índices de sustentabilidade mais compreensíveis e a lista de cidades cresceu para 100. Assim, conforme mostrado no Quadro 2, o ranking analisa três dimensões de sustentabilidade: pessoas, planeta e lucro. Estas representam, respectivamente, a sustentabilidade social, ambiental e econômica, oferecendo um indicativo da saúde e da riqueza das cidades. Dentro dessas dimensões são analisados os indicadores. Não há pesos atribuídos a cada indicador.

Quadro 2: Dimensões e indicadores do ranking Arcadis Sustainable Cities 2016

\begin{tabular}{|c|c|}
\hline Dimensões & Indicadores \\
\hline & Educação \\
& Saúde \\
Pessoas & Demografia \\
& Desigualdade de renda \\
& Poder de compra \\
& Balanço trabalho-vida \\
& Criminalidade \\
\hline Planeta & Riscos ambientais \\
& Espaços verdes \\
& Energia \\
& Poluição do ar \\
& Emissão de gases do efeito estufa \\
\hline
\end{tabular}




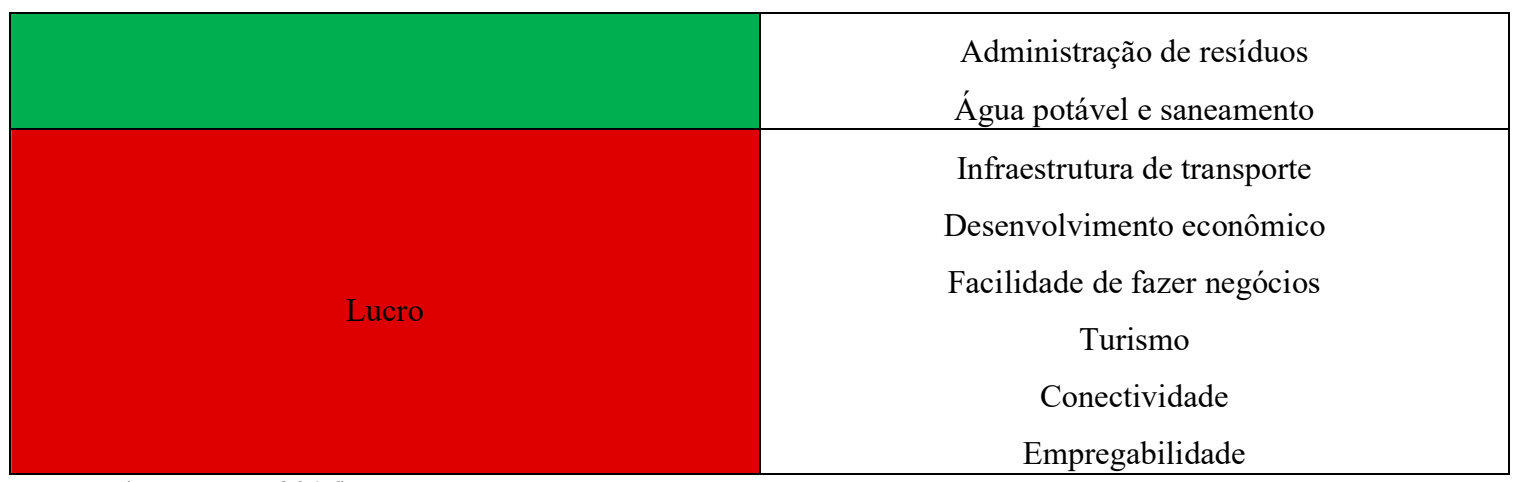

Fonte: (BATTEN, 2016)

\subsection{Relacionando os rankings}

Considerando as dimensões dos rankings, observa-se que o Arcadis apresenta três dimensões, sendo elas: pessoas, planeta e lucro. Já o ranking europeu apresenta seis dimensões, todas com "smart" como primeiro nome: economia, mobilidade, meio ambiente, pessoas, convivência e governança. Essas diferenças indicam que o ranking de cidades sustentáveis considera o tripé da sustentabilidade em suas análises. Ademais, o bem-estar social é uma das premissas para a seleção de cada um dos indicadores. Entretanto, no contexto das cidades inteligentes, mesmo que autores indiquem a tecnologia (BAKICI; ALMIRALL; WAREHAM, 2013) não há nenhuma dimensão que a especifique.

Além disso, considerando a quantidade de indicadores, mesmo com dimensões relativamente superiores, o ranking cidades inteligentes não apresenta diferenças significativas em termos de indicadores o que indica que mesmo com menos dimensões o ranking de cidades sustentáveis ainda apresenta análises robustas em termos de número de indicadores. Existe, ainda, um fator diferencial pertencente ao ranking de cidades inteligentes que são os pesos atribuídos a cada indicador. Isto produz uma distinção maior entre os rankings e entre as dimensões do próprio ranking, conferindo maior valor a umas e menor a outras (GIFFINGER; GUDRUN, 2010). O Quadro 3 ilustra a análise dos rankings de cidades inteligentes e sustentáveis.

Quadro 3: Análise dos rankings de cidades inteligentes e sustentáveis.

\begin{tabular}{|l|l|l|}
\hline \multicolumn{1}{|c|}{ Análise } & \multicolumn{1}{|c|}{ Cidades Inteligentes } & \multicolumn{1}{c|}{ Cidades sustentáveis } \\
\hline Proposta do ranking & $\begin{array}{l}\text { Mostrar como um ranking não só } \\
\text { pode fornecer o número de } \\
\text { classificação de uma cidade, mas } \\
\text { também múltiplas } \\
\text { características como base para a } \\
\text { discussão estratégica (GIFFINGER; } \\
\text { HAINDL, 2007). }\end{array}$ & $\begin{array}{l}\text { Indicar áreas de oportunidade ao invés de } \\
\text { priar uma hierarquia entre as cidades. } \\
\text { postrar as ações que as cidades tomam } \\
\text { forma econômica, ambiental e para o bem } \\
\text { de seus habitantes (BATTEN, 2016). }\end{array}$ \\
\hline Dimensões & $\begin{array}{l}\text { Economia, mobilidade, } \\
\text { ambiente, pessoas, } \\
\text { convivência e governança }\end{array}$ & Pessoas, planeta e lucro \\
\hline Indicadores & 27 & 21 \\
\hline Pesos & Sim & Não \\
\hline Número de cidades & 77 & 100 \\
\hline analisadas & &
\end{tabular}


Fonte: Elaborado pelos autores.

Em análise das convergências, a Quadro 4 apresenta a comparação entre os indicadores que apresentam dados semelhantes em suas composições. Entretanto, por vezes, certos indicadores de um dos rankings abrangem mais de um indicador do outro, como por exemplo, "Educação" em cidades sustentáveis abrange outros cinco indicadores no European Smart Cities. Por outro lado, alguns indicadores não se associam entre os rankings demonstrando medidas diferentes.

Também se tratando deste fato, o ranking de cidades sustentáveis mostra maior quantidade de indicadores envolvendo a dimensão de meio ambiente em comparação com o ranking de cidades inteligentes. O indicador "Qualidade do ar" em cidades inteligentes representa três indicadores diferentes em cidades sustentáveis.

Quadro 4: Comparação dos indicadores dos rankings

\begin{tabular}{|c|c|}
\hline Arcadis Sustainable Cities & European Smart Cities \\
\hline Educação & $\begin{array}{c}\text { Educação } \\
\text { Aprendizado ao longo da vida } \\
\text { Mente aberta } \\
\text { Consciência política } \\
\text { Instalações de educação }\end{array}$ \\
\hline Saúde & Condições de saúde \\
\hline Demografia & $\begin{array}{l}\text { Pluralidade étnica } \\
\text { Coesão social }\end{array}$ \\
\hline Criminalidade & Segurança individual \\
\hline Riscos ambientais & Consciência ecológica \\
\hline $\begin{array}{l}\text { Poluição do ar } \\
\text { Energia } \\
\text { Emissão de gases de efeito estufa }\end{array}$ & Qualidade do ar \\
\hline $\begin{array}{l}\text { Administração de resíduos } \\
\text { Água potável e saneamento } \\
\text { Espaços verdes }\end{array}$ & Administração sustentável de recursos \\
\hline Infraestrutura de transporte & $\begin{array}{l}\text { Sistema de transporte local } \\
\text { Sustentabilidade do sistema de transporte }\end{array}$ \\
\hline Desenvolvimento econômico & Produtividade \\
\hline Facilidade de fazer negócios & $\begin{array}{c}\text { Espírito inovador } \\
\text { Empreendedorismo }\end{array}$ \\
\hline Turismo & $\begin{array}{l}\text { Instalações culturais e de lazer } \\
\text { Atrações turísticas }\end{array}$ \\
\hline Conectividade & Infraestrutura - TIC \\
\hline Empregabilidade & Mercado de trabalho \\
\hline \multicolumn{2}{|c|}{ Itens individuais } \\
\hline Desigualdade de renda & Qualidade de habitação \\
\hline Capacidade de compra & Imagem da cidade \\
\hline \multirow[t]{4}{*}{ Balanço trabalho-vida } & Integração internacional \\
\hline & Acessibilidade (inter-)nacional \\
\hline & Serviços públicos e sociais \\
\hline & Administração eficiente \\
\hline
\end{tabular}

Fonte: Elaborado pelos autores. 
No quesito amplitude de análise das cidades, o ranking de cidades sustentáveis é maior em comparação ao de cidades inteligentes, uma vez que abrange um número superior de cidades do mundo todo, enquanto o ranking de cidades inteligentes restringe-se apenas às cidades europeias.

Além disso, o ranking europeu divulgou a classificação apenas para cidades de cem mil a quinhentos mil habitantes, enquanto o outro ranking não apresenta essa restrição.

\section{Conclusões}

A partir dos dados descritos sobre cada um dos rankings apresentados por este trabalho, pôde-se perceber que ambos apresentam convergências de informações no que tange seus indicadores. A preocupação, dos dois rankings, com a saúde econômica, ambiental e populacional das cidades é identificada, entretanto o foco de cada um é divergente em conformidade com suas propostas. O ranking Arcadis Sustainable Cities demonstrou um foco maior, como era de se esperar, nos itens que têm maior relação com a saúde ambiental, dispondo de maior quantidade de indicadores ligados à preservação do meio ambiente e do bem-estar populacional. Já o ranking European Smart Cities tem menor quantidade de indicadores na parte ambiental, entretanto focalizou-os mais na parte de convivência, economia e tecnologia.

\section{Referências}

AHVENNIEMI, $\mathrm{H}$. et al. What are the differences between sustainable and smart cities? Cities, v. 60, p. 234-245, 2017.

ALBINO, V.; BERARDI, U.; DANGELICO, R. M. Smart Cities : Definitions , Dimensions , Performance, and Initiatives. n. February, p. 37-41, 2015.

BAKICI, T.; ALMIRALL, E.; WAREHAM, J. A Smart City Initiative: The Case of Barcelona. Journal of the Knowledge Economy, v. 4, n. 2, p. 135-148, 2013.

BATTEN, J. Sustainable Cities Index 2016. Arcadis Global, p. 10, 2016.

BRUNDTLAND, G. H. Our Common Future: Report of the World Commission on Environment and Development. United Nations Commission, v. 4, n. 1, p. 300, 1987.

BULKELEY, H.; BETSILL, M. M. Rethinking Sustainable Cities: Multilevel Governance and the "Urban" Politics of Climate Change Rethinking Sustainable Cities: Multilevel Governance and the "Urban" Politics of Climate Change. Environmental Politics, v. 14, n. February, p. 42-63, 2005.

CARRETERO, M. I. Clay minerals and their beneficial effects upon human health. A review. Applied Clay Science, v. 21, n. 3-4, p. 155-163, 2002.

ELIAS, S.; KROGSTIE, J. Smart sustainable cities of the future: An extensive interdisciplinary literature review. Sustainable Cities and Society, v. 31, p. 183-212, 2017.

EUROPEAN COMMISSION. Cities of tomorrow. [s.l: s.n.].

GIFFINGER, R.; GUDRUN, H. Smart cities ranking: an effective instrument for the positioning of the cities? ACE: Architecture, City and Environment, v. 4, n. 12, p. 7-26, 2010 .

GIFFINGER, R.; HAINDL, G. Smart Cities Ranking: an Effective Instrument for the 
Positioning of Cities? p. 703-714, 2007.

GIL, A. C. Como elaborar projetos de pesquisa. [s.l: s.n.]. v. 214

GOODLAND, R.; DALY, H. Environmental Sustainability: Universal and Negotiable. Ecological applications, v. 6, n. 4, p. 1002-1017, 1996.

HIREMATH, R. B. et al. Indicator-based urban sustainability-A review. Energy for Sustainable Development, v. 17, n. 6, p. 555-563, 2013.

HOLDEN, E.; LINNERUD, K.; BANISTER, D. The Imperatives of Sustainable Development. Sustainable Development, v. 25, n. 3, p. 213-226, 2017.

JESSOP, B. An Entrepreneurial City in Action: Hong Kong's Emerging Strategies in and for (inter)Urban Competition. Urban Studies, v. 37, n. 12, p. 2315-2335, 2000.

KANTER, R. M.; LITOW, S. S. Informed and Interconnected: A Manifesto for Smarter Cities Informed and Interconnected : A Manifesto for Smarter Cities $\square .2009$.

KENNEDY, C.; CUDDIHY, J.; ENGEL-YAN, J. of Cities. v. 11, n. 2, 2007.

KUHLMAN, T.; FARRINGTON, J. What is sustainability? Sustainability, v. 2, n. 11, p. 3436-3448, 2010.

MEIJERING, J. V.; KERN, K.; TOBI, H. Identifying the methodological characteristics of European green city rankings. Ecological Indicators, v. 43, p. 132-142, 2014.

NAM, T.; PARDO, T. A. Smart city as urban innovation: Focusing on management, policy, and context. Proceedings of the 5th International Conference on Theory and Practice of Electronic Governance - ICEGOV'11, p. 185, 2011.

RAI, P. T. Townships for Sustainable Cities. v. 37, p. 417-426, 2012.

SCIENCE FOR ENVIRONMENT POLICY. Indicators for sustainable cities. In-depth Report, n. 12, p. 1-24, 2015.

SUZUKI, H. et al. Eco Cities. [s.l: s.n.].

TELOS; DAGEVOS, J. F. L. M. M.; ZOETEMAN, K. Integrated Sustainability Monitoring of 58 EU-Cities. 2015.

UNITED NATIONS, DEPARTMENT OF ECONOMIC AND SOCIAL AFFAIRS, P. D. World Urbanization Prospects. United Nations, v. 12, p. 32, 2014.

EUROPEAN SMART CITIES. European Smart Cities. Disponível em: <http://www.smartcities.eu/?cid=2\&ver=4>. Acesso em 06 jul. 2017. 